\title{
Evaluación de la aceptación del distintivo "Q" ofertado por el GAD provincial Santo Domingo de los Tsáchilas, en los centros turísticos de la provincia, año 2017
}

\section{DIEGO FABRICIO DÉFAZ GONZÁLEZ*, CRISTHIAN GERMÁN RODRÍGUEZ BONNILLA, VERÓNICA ANDREA TOAPANTA PAUTA, BÉLGICA PAMELA LÓPEZ CEDEÑO}

Instituto Superior Tecnológico Tsáchila

Av. Galo Luzuriaga y calle B, Santo Domingo, Ecuado

*dfdefazg@gmail.com

\section{RESUMEN}

El objetivo de este estudio fue determinar la oferta, demanda y nivel de aceptación del distintivo " $Q$ " ofertado por el GAD Provincial Santo Domingo de los Tsáchilas, para centros turísticos de la provincia Santo Domingo de los Tsáchilas, el cual formó parte de un plan de marketing que desarrolló estrategias para difundir el distintivo antes mencionado; que propone mejorar la calidad de los servicios prestados en el área del turismo. Consecuentemente aplicando conocimientos de estadística descriptiva e inferencial se sustentó la viabilidad para el plan antes mencionado. El estudio fue realizado a
162 establecimientos como muestra representativa de la población en estudio; seleccionados bajo un muestreo probabilístico, mismos que estaban inscritos en el catastro de instituciones turísticas a cargo del GAD Provincial de Santo Domingo de los Tsáchilas, segmentados por actividad, subtipo de actividad y categoría; determinando finalmente razones por las cuales se debería implantar un distintivo de Calidad Turística en la provincia que vincula los objetivos del Plan Nacional del Buen Vivir y el Plan de Desarrollo de la Provincia Santo Domingo 2025 (Plan Estratégico), con el fín de integrar de manera satisfactoria normas de calidad que cada vez son prioridad en las empresas locales, nacionales y extranjeras.

Palabras clave: Centros turísticos, calidad, distintivo $Q$, oferta, demanda.

\section{INTRODUCCIÓN}

Ecuador es una región rica en biodiversidad que está creciendo rápidamente, hoy es un país en vía de desarrollo con grandes objetivos en los ámbitos académico, industrial, económico, turístico, entre otros que se encuentran plasmados en el Plan Nacional de Desarrollo. 
Ecuador alberga a 15.74 millones de habitantes los cuales día a día hacen que este sea un mejor país. En ese mismo sentido, el Estado recibió en julio del 2014 a 904.249 turistas extranjeros, en relación al año 2013, esta cifra representa un incremento del 14\%. Este resultado se debe a campañas nacionales en favor del turismo. Retrospectivamente, en el año 2010 se desarrolló una estrategia comunicacional con el fin de convertir al Ecuador en potencia turística, basándose en una filosofía andina como el "Sumak Kawsay" entre otros elementos tomados de la realidad, dando origen a "Ecuador ama la vida", con la misión de que las personas puedan identificarse en un contexto global.

Posterior a ello se lanza una nueva campaña el 1 de abril del 2014, con el fin de promocionar al Ecuador como destino turístico a través del lema "All You Need is Ecuador", que consistía en implantar letras de 6 metros de alto instaladas en plazas, parques y lugares emblemáticos, en 19 ciudades del mundo, 7 de ellas de Ecuador. Impactando a más de 450 millones de personas en el mundo, pues se difunde en español, inglés, portugués, alemán y francés.

Dentro de este magnífico país, se encuentra la provincia de Santo domingo de los Tsáchilas, en tiempos de la colonia se conocía como la provincia de los Yumbos. En ellas habitaban las etnias Tsáchilas, Yumbos y Chachis. Hoy en día cuenta con una extensión de $3778.57 \mathrm{~km} 2$, es el sector geográfico donde convergen las provincias de Esmeraldas, Manabí, Guayas, Los Ríos y Cotopaxi. Por ello se hace necesario mencionar, que Santo Domingo nace como Parroquia el 29 de mayo de 1861, posterior a ello el 3 de julio del año 1967, mediante registro oficial (R.O.) No.161 es nombrado Cantón, finalmente para el 6 de noviembre del 2007 mediante R.O. No.205 Suplemento, es nombrada Provincia; con una población de 368.013 personas según INEC hasta el 2010. Dentro de este territorio, existen hasta la presente fecha 305 centros turísticos que contribuyen al desarrollo económico de la provincia y país; donde recibe 31142 turistas extranjeros en promedio según datos del año 2014.

No obstante, si bien ha existido un auge del turismo en estos últimos años las empresas o centros turísticos registrados en localidad no están presentando en su mayoría una atención de calidad, donde se cumplan con las exigencias internacionales y expectativas de los turistas extranjeros. Si bien nos son todos los centros que presentan esta problemática, es un efecto que se podría mejorar a través de ciertas medidas que impulsen un desarrollo y mejoramiento en calidad turística.

Por tal razón, la Fundación Conservación \& Desarrollo (C\&D) ha creado la norma Smart Voyager Express con el fin de brindar una asistencia técnica a las propietarios y colaboradores de operaciones turísticas y encaminarlos hacia la implementación de buenas prácticas en turismo sostenible. Esta norma abarca ámbitos ambientales, sociales, empresariales y por su puesto la prestación del servicio en hospedaje, alimentos y bebidas encaminados a un alto nivel de calidad. En igual forma, la Empresa Pública Metropolitana de Gestión de Destino Turístico - Quito Turismo, vio esta gran oportunidad de usar la norma Smart Voyager Express como base para la creación del Distintivo " $Q$ ", con el fin de promover la implementación de sistemas de gestión de turismo sostenible, calidad y profesionalización del equipo humano en los establecimientos turísticos del Distrito Metropolitano de Quito, para el mejoramiento de la competitividad del destino Quito, obteniendo hasta la presente fecha resultados satisfactorios que invitan a seguir trabajando en el promover el Distintivo " $Q$ " de Quality que significa "Calidad"; invitando a los Gobiernos Descentralizados de la Provincia de Santo Domingo a replicar un distintivo de Calidad Turística en su localidad. 


\section{MARCO REFERENCIAL}

\subsection{Norma Smart Voyaver Xpress}

"La norma Smart Voyaver Xpress brinda asistencia técnica a los propietarios y colaboradores de operaciones turísticas y encaminarlos hacia la implementación de buenas prácticas en turismo sostenible" (Conservación y Desarrollo.web, 2015).

"El Alcance de la norma incluye el cumplimiento de parámetros ambientales, sociales, empresariales y sobre todo de un alto nivel de calidad en la prestación del servicio en hospedaje y alimentos \& bebidas" (Conservación y Desarrollo.web, 2015).

Los beneficios que presentan las empresas turísticas con distintivo de calidad "Smart Voyager Express" forman parte de una gran comunidad que trabaja por el turismo sostenible y su mercadeo de manera directa y exitosa. La empresa con distintivo de calidad recibirá capacitación, promoción, intercambio de información, además de un cuidadoso acompañamiento para implementar procesos sostenibles. (Conservación y Desarrollo.web, 2015)

Smart Voyaver Xpress es una norma que busca mejorar el servicio de turismo y mercadeo hacia una cosmovisión de conservación de los recursos naturales tales como flora y fauna, además de buscar siempre la calidad a través de buenas prácticas turísticas que son impartidas en cursos, boletines que les permite mantenerse innovando comprometidas con el desarrollo sostenible.

\subsection{Distintivo " $Q$ "}

Es un reconocimiento que otorga el Municipio a través de la Empresa Pública Metropolitana de Gestión de Destino Turístico Quito Turismo a los establecimientos turísticos que han implementado sistemas de gestión de turismo sostenible y calidad. El distintivo " $Q$ " proviene de Qualitas que en latín significa Calidad. (Quito Turismo. web, 2015)

"Promover la implementación de sistemas de gestión de turismo sostenible, calidad y profesionalización del equipo humano en los establecimientos turísticos del Distrito Metropolitano de Quito para el mejoramiento de la competitividad del destino Quito" (Quito Turismo. web, 2015).

"Incluye el cumplimiento de parámetros ambientales, sociales, empresariales y de calidad" (Quito Turismo.web, 2015).

- Otorgamiento de asistencia técnica especializada y capacitación para elevar la calidad de la prestación de los servicios turísticos y la competitividad del destino, en base a herramienta de gestión de turismo sostenible.

- Mejoramiento de la calidad de servicios y productos comerciales de las empresas, para fomentar el crecimiento de la demanda turística.

- Profesionalización del equipo humano en los establecimientos. (Quito Turismo.web, 2015)

Distintivo $Q$ es una norma creada por la empresa Quito Turismo con el fin de mejorar la calidad turística en el Distrito Metropolitano de Quito, manteniendo estándares ajustados a la realidad turística de la 
localidad que engloban el ecosistema, sociedad y empresas; inspirada en la norma Smart Voyager Xpress mantiene un seguimiento de las empresas que forman parte de esta distinción a la calidad turística con asistencia técnica y capacitaciones que aseguran la calidad de los servicios turísticos.

\subsection{Turismo}

"Conjunto de actividades que realizan las personas durante sus desplazamientos y estancias en distintos lugares diferentes al de su entorno habitual, por un periodo de tiempo consecutivo menos a un año, con fines de ocio, negocios y otros motivos" (Cabarcas, 201 1, p. 2).

Actividad que realizan las personas que se movilizan fuera de su entorno cotidiano, por un tiempo determinado que no sea mayor a un año, en la localidad el turismo se ve reflejado en actividades como el conocer las reservas o parques forestales, balnearios y las comunas de la cultura autóctona de la provincia siendo éstos los Tsáchilas.

\section{METODOLOGÍA DE LA INVESTIGACIÓN}

\subsection{Enfoque / Tipo de investigación}

"El Enfoque Cuantitativo obtiene información que poder medirse de forma objetiva y generalizarse a una población" (Alarcón, et al., 2014 , p. 71).

Se trabaió desde el enfoque cuantitativo, debido a que permitió analizar datos, los cuales fueron medidos y cuantificados, además de que este enfoque, se sirve de múltiples métodos y técnicas como la estadística, que fue muy necesaria para poder realizar las tabulaciones, muestreos, etc.

\subsection{Tipo de investigación}

"La Investigación descriptiva busca especificar propiedades, características y rasgos importantes de cualquier fenómeno que se analice. Describe tendencias de un grupo o población" (Hernández, Fernández, \& Baptista, 2010, p. 80).

Este tipo de investigación se aplicó porque en su momento, se limitó únicamente a observar y describir lo que acontecía en el entorno dentro del marco de la presente investigación, los cuales ayudados en su momento de la estadística pudieron ser visualizados para una descripción.

"La Investigación explorativa se realizan cuando el objetivo es examinar un tema o problema de investigación poco estudiado, del cual se tiene muchas dudas o no se ha abordado antes" (Hernández, Fernández, \& Baptista, 2010, p. 79).

Se aplicó la investigación exploratoria, puesto que es un tema nuevo que no se ha estudiado antes a nivel académico y en caso de haber información es limitada, lo cual dificulta la investigación, lo que exigía realizar una investigación en búsqueda de información que facilite la comprensión del problema planteado.

"La investigación explicativa está dirigido a responder por las causas de los eventos físicos o sociales. Se enfocan en explicar porque ocurrió un fenómeno y en qué condiciones se manifiestan, o porque se relacionan dos o más variables" (Hernández, Fernández, \& Baptista, 2010, p. 85).

A través de este tipo de investigación, se explicaron las razones de los efectos encontrados al iniciar el plan de investigación y durante el proceso del mismo, se pudo identificar las distintas causas que debían ser atendidas y corregidas para evitar efectos no deseados. 
Evaluación de la aceptación del distintivo " $Q$ "

Cristhian Rodríguez B., Verónica Toapanta P., Bélgica López C. • VÍNCULOS-ESPE (2019) VOL. 4, No.2:22-29

"La Investigacón correlacional tiene como finalidad conocer la relación o grado de asociación que exista entre dos o más conceptos, categorías o variables en un contexto en particular" (Hernández, Fernández, \& Baptista, 2010, p. 81).

Mediante este tipo de investigación, se llegó a establecer relaciones entre los actores y factores del estudio y cómo éstos influyen sobre el otro, lo cual ayudó a poder aplicar estrategias eficaces, teniendo en cuenta que éstas podían influir en otras.

\section{DESARROLLO}

En conformidad con la información obtenida de las 162 encuestas realizadas en la provincia de Santo Domingo de los Tsáchilas a propietarios y/o administradores de los diferentes establecimientos turísticos tales como: hoteles, moteles, bares, cafeterías, restaurantes y centros de recreación turística de las zonas urbanas y rurales; la tabulación de los datos se trabaió en el software IBM SPSS Statstics Versión 21 , determinando con el $93.83 \%$ de la población objetivo el desconocimiento del distintivo $Q$ como norma de calidad, pero a pesar de ello existe una aceptación al mismo siempre que mejore, asista y controle la calidad de los servicios turísticos prestados con el fin de contribuir al desarrollo sostenible del comercio y turismo provincial, valores reflejados con el $64.81 \%$ como medianamente probables y con el $33.33 \%$ muy probables en su adquisición.

Por consiguiente, dentro de un mercado que con el $65.43 \%$ de puntuación, actualmente se considera moderadamente competitivo que presta servicios catalogados con el $84.57 \%$ de buena calidad, confían en el que esta iniciativa puede llegar a ser muy útil si se implanta responsablemente; atendiendo a las consideraciones antes expuestas, se concluye la viabilidad para ejecutar la propuesta de difundir el distintivo como norma de calidad turística respaldada por el GAD Provincial Santo Domingo de los Tsáchilas, organismo que cuenta con el $96.91 \%$ credibilidad y aceptación por parte de la población objetivo.
Tabla 1

Informe final

\begin{tabular}{|c|c|c|c|c|c|}
\hline Pregunta 3 & Criterios & & Frecuencia & $\begin{array}{c}\text { Porcentaje } \\
\quad \%\end{array}$ & $\begin{array}{c}\text { Porcentaje } \\
\text { válido \% }\end{array}$ \\
\hline \multirow{4}{*}{$\begin{array}{l}\text { Ha escuchado del } \\
\text { distintivo } Q\end{array}$} & Válidos & $\begin{array}{l}\text { Nunca he } \\
\text { escuchado de él }\end{array}$ & 152 & 93,83 & 93,83 \\
\hline & & $\begin{array}{l}\text { He escuchado } \\
\text { sobre él pero } \\
\text { nunca he } \\
\text { investigado }\end{array}$ & 8 & 4,94 & 4,94 \\
\hline & & $\begin{array}{l}\text { Si conozco sobre } \\
\text { el distintivo } Q\end{array}$ & 2 & 1,23 & 1,23 \\
\hline & & Total & 162 & 100,00 & 100,00 \\
\hline Pregunta 7 & Criterios & & Frecuencia & $\begin{array}{c}\text { Porcentaje } \\
\quad \%\end{array}$ & $\begin{array}{l}\text { Porcentaje } \\
\text { válido \% }\end{array}$ \\
\hline \multirow{4}{*}{$\begin{array}{l}\text { Cómo calificaría la } \\
\text { contribución de la } \\
\text { gestión de calidad } \\
\text { dentro de las } \\
\text { organizaciones o } \\
\text { empresas }\end{array}$} & Válidos & Baja contribución & 3 & 1,85 & 1,85 \\
\hline & & $\begin{array}{l}\text { Mediana } \\
\text { contribución }\end{array}$ & 130 & 80,25 & 80,25 \\
\hline & & Alta contribución & 29 & 17,90 & 17,90 \\
\hline & & Total & 162 & 100,00 & 100,00 \\
\hline Pregunta 9 & Criterios & & Frecuencia & $\begin{array}{c}\text { Porcentaje } \\
\%\end{array}$ & $\begin{array}{c}\text { Porcentaje } \\
\text { válido \% }\end{array}$ \\
\hline \multirow{4}{*}{$\begin{array}{l}\text { SI estuviera en vigencia } \\
\text { el distintivo Q, cuál es } \\
\text { la probabilidad de que } \\
\text { se organización o } \\
\text { empresa participe para } \\
\text { su adquisición }\end{array}$} & Válidos & Baja probabilidad & 3 & 1,85 & 1,85 \\
\hline & & $\begin{array}{l}\text { Mediana } \\
\text { probabilidad }\end{array}$ & 105 & 64,81 & 64,81 \\
\hline & & Alta probabilidad & 54 & 33,33 & 33,33 \\
\hline & & Total & 162 & 100,00 & 100,00 \\
\hline Pregunta 10 & Criterios & & Frecuencia & $\begin{array}{c}\text { Porcentaje } \\
\%\end{array}$ & $\begin{array}{l}\text { Porcentaje } \\
\text { válido \% }\end{array}$ \\
\hline \multirow{5}{*}{$\begin{array}{c}\text { De los múltiples } \\
\text { ámbitos en los que ese } \\
\text { ofrece mejorar el } \\
\text { servicio de calidad, cuál } \\
\text { de estos le interesaría } \\
\text { más }\end{array}$} & Válidos & $\begin{array}{l}\text { Gestión } \\
\text { administrativa }\end{array}$ & 7 & 4,32 & 4,32 \\
\hline & & $\begin{array}{l}\text { Responsabilidad } \\
\text { social } \\
\text { empresarial }\end{array}$ & 11 & 6,79 & 6,79 \\
\hline & & $\begin{array}{l}\text { Infraestructura y } \\
\text { equipamiento }\end{array}$ & 31 & 19,14 & 19,14 \\
\hline & & $\begin{array}{l}\text { Calidad del } \\
\text { servicio y } \\
\text { atención al cliente }\end{array}$ & 113 & 69,75 & 69,75 \\
\hline & & Total & 162 & 100,00 & 100,00 \\
\hline
\end{tabular}

Fuente: Investigación de campo. (2017). Santo Domingo. 


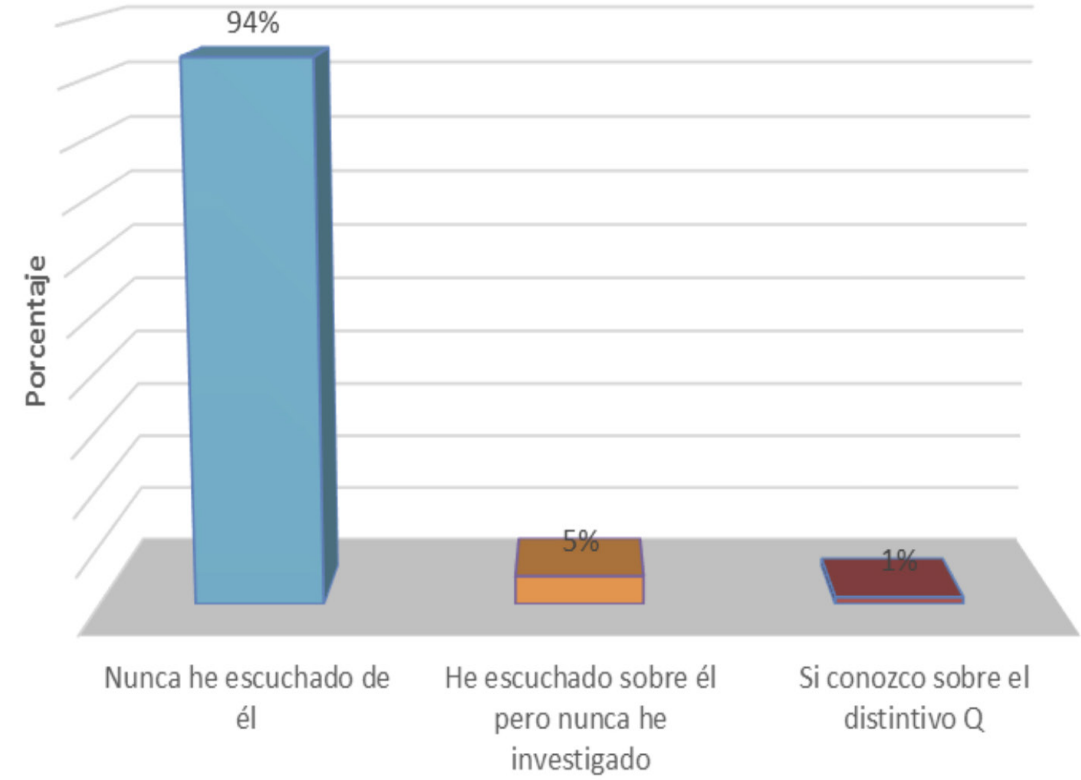

Figura 1. Conocimiento acerca del distintivo $Q$ Fuente: Investigación de campo. (2017). Santo Domingo.

Con el fin de reflejar el grado de conocimientos acerca de esta norma y proyectar el esfuerzo que se debe hacer para cambiar la realidad de los datos obtenidos, esta pregunta determina el porcentaje o cantidad de personas que conocen acerca del distintivo Q, dentro de la provincia de Santo Domingo de los Tsáchilas. Según la investigación realizada, se determina que el $94 \%$ de la población en estudio desconoce acerca del distintivo $Q$, lo cual propone que se trabaje en publicidad para comunicar los objetivos y beneficios del distintivo, permitiendo informar de la manera más conveniente y evitando malinterpretaciones que puedan perjudicar el proyecto.

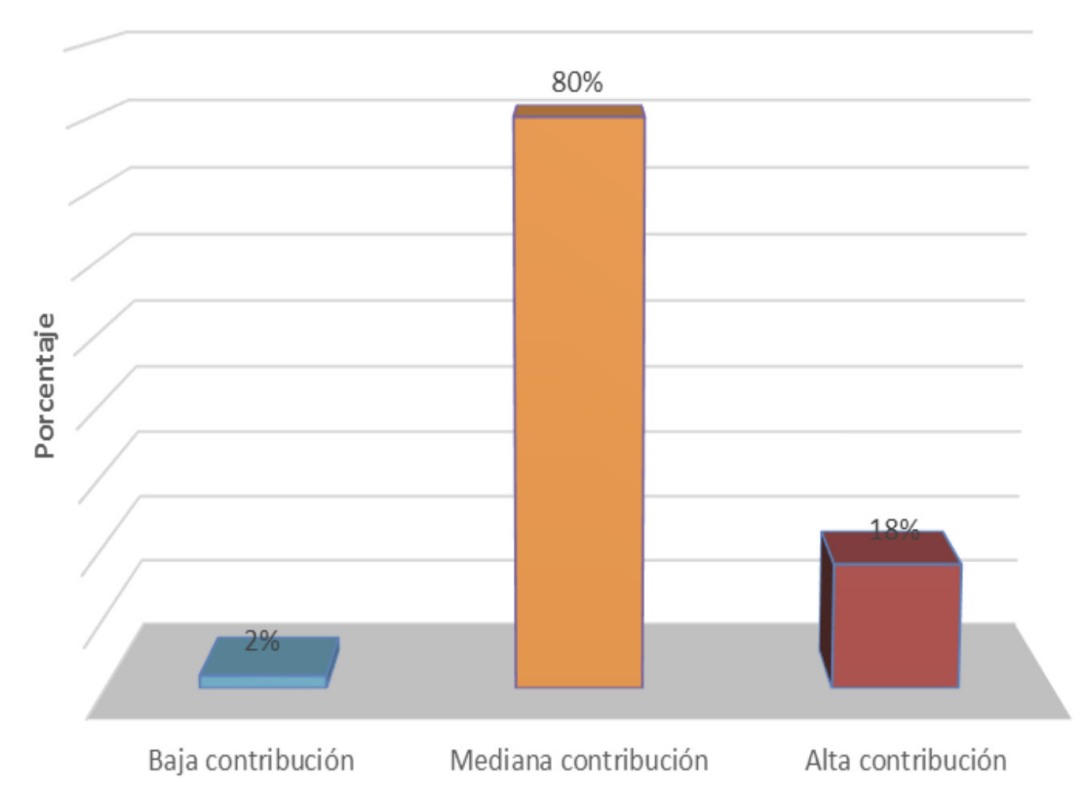

Figura 2. Contribución de la gestión de calidad Fuente: Investigación de campo. (2017). Santo Domingo.

Conocer la contribución que tiene la gestión de calidad dentro de las instituciones turísticas de la provincia de Santo Domingo de los Tsáchilas, es importante para el proyecto con el fin de establecer acciones que permitan fortalecer y/o mejorar realidades adversas al proyecto. El $80 \%$ de la población considera que la gestión de calidad tiene una mediana contribución dentro de las organizaciones, seguido de un $18 \%$ que considera que es alta la contribución y en contraste con las anteriores existe un $2 \%$ que considera que es baja, lo cual encamina a este proyecto en buen horizonte debido que el distintivo $Q$ fomenta la gestión de calidad turística dentro de las instituciones. 


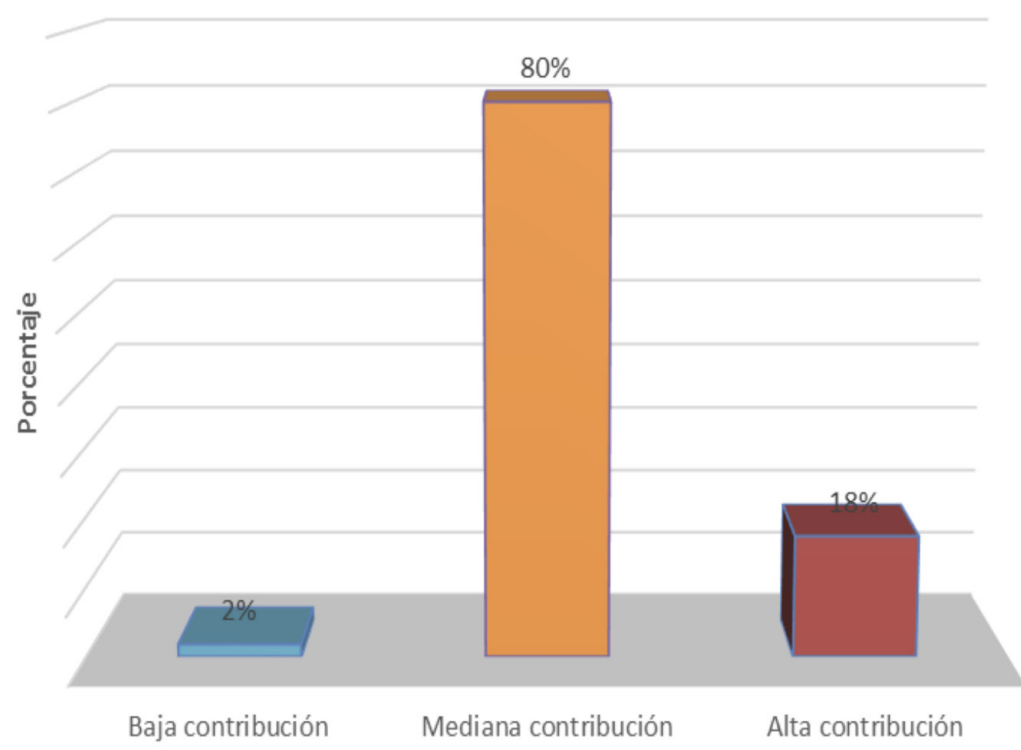

Figura 3. Probabilidad para adquisición del distintivo $Q$ Fuente: Investigación de campo. (2017). Santo Domingo.

En cuanto a la probabilidad de participación para obtener el distintivo a través del GAD provincial Santo Domingo de los Tsáchilas como único oferente, cuantas instituciones serían las que participen en el programa de calidad turística (Distintivo Q), teniendo en consideración la pregunta anterior en la que el total de encuestados afirmó que sí lo recomendarían. Partiendo de los supuestos anteriores, se determina que el $65 \%$ de la población afirma tener una mediana probabilidad considerándolos inseguros, lo cual nos indica que se debe trabajar con estrategia para captar este porcentaje, seguido con el $33 \%$ de la población correspondiente a una alta probabilidad podemos considerarlos seguros para la participación en obtener el distintivo $Q$ y en contraste con una probabilidad baja que se considera nulos tenemos el $2 \%$ de la población sujeta al estudio de mercado.

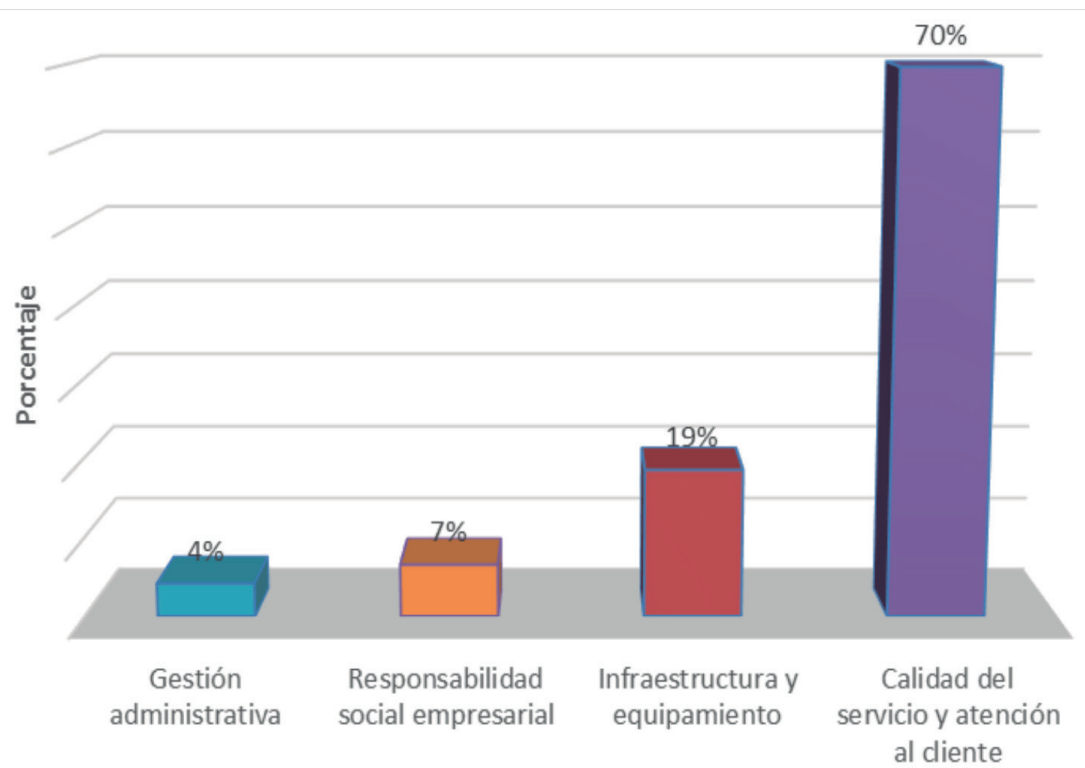

Figura 4. Ámbitos del distintivo $Q$

Fuente: Investigación de campo. (2017). Santo Domingo.

Referente a las áreas que ofrece asistencia el distintivo $Q$, cuáles serían las que primordialmente son de interés a las empresas u organizaciones, pudiendo determinar así la carencia de las instituciones turísticas y proyectar acciones encaminadas en atender estas necesidades latentes.

En consecuencia a lo anteriormente expuesto, se determina que el sector turístico está interesado en la asistencia para mejorar la calidad del servicio y atención al cliente, lo cual se ve reflejado con el $70 \%$ de selección sobre los demás criterios propuestos; se ha determinado que el servicio y atención actual mayoritariamente se lo realiza de manera empírica y tradicional debido a que los negocios son familiares. 


\section{CONCLUSIONES}

Se ha encontrado que casi la totalidad de los encuestados desconocen acerca del distintivo $Q$ y los beneficios que ofrece a las instituciones turísticas, a pesar de que en Quito se lleve desarrollando por más de 3 años esta iniciativa; teniendo en consideración que la mayoría de los encuestados tiene acceso a Internet y redes sociales.

A través del análisis inferencial se ha determinado la relación y asociación de variables como: edad, probabilidad de adquisición para el distintivo $Q$, competitividad del mercado turístico, entre otras; que confirman la vialidad del presente proyecto.

\section{REFERENCIAS BIBLIOGRÁFICAS}

Alarcón, M., Cordente, M., Gómez, M., Blázquez, J., Millán, A., Díaz , E., \& Consuegra, D. (2014). Investigación de mercados. Madrid: ESIC Editorial.

Cabarcas, N. (2011). Administración de servicios turísticos. Bogotá: Ediciiones de la $\mathrm{U}$.
Conservación y Desarrollo.web. (25 de 05 de 2017). Certificación para la Sostenibilidad Turística Smart Voyager. Obtenido de http://www. smartvoyager.org/

Hair, J., Bush, R., \& Ortinav, D. (2010). Investigación de mercados en un ambiente de información digital. México: Mc Graw Hill.

Hernández , R., Fernández, C., \& Baptista, P. (2010). Metodología de la investigación (5ta ed.). México: Mc Graw Hill.

Quito Turismo.web. (25 de 05 de 2017). Empresa Pública Metropolitana de Gestión de Destino Turístico. Obtenido de http://www.quito-turismo. gob.ec/index.php/destacados/3-distintivo-q

Sarmiento, B., \& Fernández, F. (2014). Estadística descriptiva. Bogotá: Ediciones de la U.

Cámara de comercio Sto. Dgo. (24 de 01 de 2016). Cámara de Comercio Sto Dgo. Obtenido de http://www.camaracomerciostodgo.com/

Smartvoyager.org. (25 de 05 de 2015). Certificación para la Sostenibilidad Turística Smart Voyager. Obtenido de http://www.smartvoyager.org/

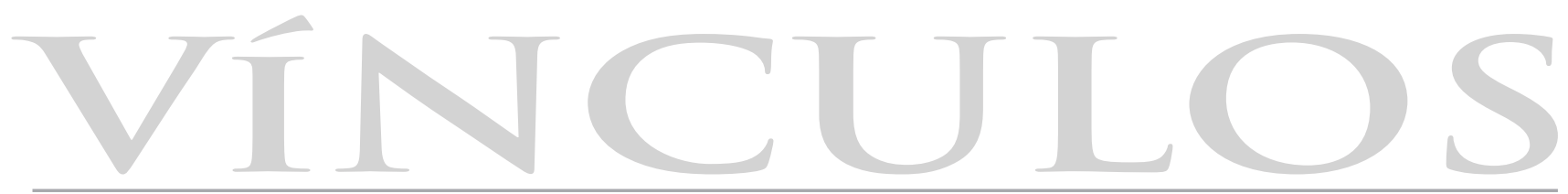

U N I V E R I D A D D E L A S F U ER Z A S A M A D A S E P E 